\title{
Étude de l'auto-évaluation de la qualité de parole et du ressenti (SHI) après glossectomie partielle ou totale
}

\author{
Hasna Zaouali ${ }^{1}$, Béatrice Vaxelaire ${ }^{1}$, Christian Debry ${ }^{2}$, Rudolph Sock $^{1 \& 3}$ \\ ${ }^{1}$ U.R. 1339-Linguistique, Langues et Parole (LiLPa) -ER Parole et Cognition \\ Institut de Phonétique de Strasbourg (IPS) - Université de Strasbourg \\ 22 rue Descartes - 67084 Strasbourg- Cedex, France. \\ ${ }^{2}$ Service O.R.L. - Hôpitaux Universitaires de Strasbourg \\ 1 av. Molière - 67098 Strasbourg - Cedex, France. \\ ${ }^{3}$ LICOLAB - Université Pavla Jozefa Safarika, Faculté des Lettres Košice - Slovaquie. \\ 041 80, Šrobárova 1014/2, 04001 Košice, Slovakia
}

\section{hasnazaouali@live.fr}

\begin{abstract}
Résumé : Étude de l'auto-évaluation de la qualité de parole et du ressenti (SHI) après glossectomie partielle ou totale.

Cette étude consiste en une analyse des réponses recueillies auprès de patients glossectomisés après la passation du questionnaire d'auto-évaluation de la parole et du ressenti (Speech Handicap Index ou SHI) de (Rinkel \& coll,.2008), traduit en français par Degroote et al. (2012) et adapté du VHI (Voice Handicap Index de Jacobson et al., 1997). Le SHI est un outil d'évaluation du degré du handicap provoqué par les troubles de la parole. Il est constitué d'une échelle d'autoévaluation cotée sur 120 points et comporte 30 items. Ce questionnaire est constitué de deux sous échelles dont une consacrée à l'aspect Psycho-sociale(Ps) et l'autre à l'aspect Parole (s). L'objectif d'évaluer le handicap d'un patient glossectomisé est de mieux comprendre les dysfonctionnements sur le plan linguistique pour une meilleure prise en charge thérapeutique.

Nous avons examiné, à travers des phases de passations pré et post-chirurgicales, les réponses collectées auprès de chaque patient. Une analyse statistique est présentée afin de rendre compte de la sévérité du handicap entre les phases de passations du questionnaire d'auto évaluation de la parole et du ressenti.
\end{abstract}

\section{Abstract: Study of speech quality self-assessment (SHI) after partial or total glossectomy.}

This study consists of an analysis of responses collected from glossectomised patients after completing the Speech Handicap Index questionnaire (SHI) elaborated by (Rinkel \& al., 2008), translated into French by Degroote et al. (2012) and adapted from the VHI version (Voice Handicap Index by Jacobson et al., 1997). SHI is a tool for assessing the degree of handicap caused by speech disorders. It consists of a self-assessment scale rated on 120 points and includes 30 items. This questionnaire comprises two sub-scales, one devoted to the Psychosocial (Ps) aspect and the other to the speech (s) aspect. The objective of assessing a glossectomised patient's handicap is to better understand linguistic dysfunctions in order to 
design appropriate therapeutic treatments. We examined all responses collected from each patient, throughout the different pre- and post-surgical phases.

Statistical analyses were carried out to account for the severity of the handicap between the different self-assessment phases of speech and self-esteem in the questionnaire. 


\section{Introduction}

La qualité de vie après un cancer des voies aérodigestives supérieures (VADS) varie d'un individu à un autre; elle est évaluée sur plusieurs phases, de l'annonce du diagnostic, pendant les traitements chirurgicaux et complémentaires et même après un an ou plus. En effet, malgré les répercussions que les traitements peuvent engendrer dans les VADS, le patient tente de s'adapter à sa nouvelle vie. Il existe peu d'études sur la qualité de vie après un cancer des VADS. Cependant, la plupart de ces études notent une perturbation sérieuse de la parole et de la déglutition pendant le traitement, ainsi qu'une détérioration de la dimension psychosociale du patient et de son insertion dans la vie de tous les jours.

Une perturbation est perçue après un an pour certains patients mais ce n'est pas toujours le cas pour d'autres (Gritz et al., 1999 ; List AM et al., 1999). Durant les 6 premiers mois, on constate une dégradation remarquable de la qualité de vie du patient suite à la fatigue, la douleur et la xérostomie. D'autres travaux évoquent la dégradation de la qualité de vie du patient résultant de son état psychique et la dépression ; cela concerne des patients dans des stades tumoraux assez avancés T4 (Hammerlid et al., 1999). D'autres études insistent sur cette conséquence (impact psychique) à long terme ; même après plusieurs années, des patients ont été retrouvés marqués négativement par cette expérience malgré le temps passé (Bjordal et al., 1994).

Ce travail de recherche s'insère dans le cadre général des perturbations et des réajustements (Sock, 1998 ; Vaxelaire, 2006) en production et en perception de la parole, et dans celui du cadre spécifique des problématiques liées aux chirurgies de la cavité orale, et plus précisément des chirurgies de la langue. Cette opération peut générer de fortes dépréciations des trois fonctions de base de la vie humaine, à savoir mastiquer, avaler, mais également des difficultés durables au niveau de la production-perception de la parole (Acher et al., 2014). On remarque également des effets sur le plan psychosocial, et de ce fait, la santé, la qualité de vie et la qualité de la parole des patients s'en trouvent remarquablement altérées (Calmet-Smadja \& Crevier-Buchman, 2003 ; Savariaux et al., 2001).

Les interventions chirurgicales de la cavité buccale engendrent des séquelles fonctionnelles souvent importantes et qui ont un impact direct sur le plan psychosocial et de l'expression orale. L'exérèse réalisée affecte toujours la mobilité linguale. Apprécier les qualités perceptives en mesurant précisément le handicap lié aux troubles de la parole permettrait de mieux les comprendre, et donc de mieux prendre en charge les patients concernés. Nous avons adopté une approche interdisciplinaire avec des analyses multiparamétriques élaborées par des cliniciens et des phonéticiens expérimentateurs. La production des patients ayant subi une ablation partielle ou totale de la langue a été enregistrée aux services O.R.L de différents établissements situés en Alsace.

Ce travail vise, à partir de la passation du dispositif d'auto-évaluation de la parole et du ressenti, le Speech Handicap Index (SHI), introduit dans le champ de la pratique clinique et expérimental, à tester la sensibilité de ce dispositif aux changements morphologiques provoqués par la glossectomie. Il s'agit d'évaluer la capacité du dispositif à objectiver des variations perceptibles sur le plan subjectif, et d'observer spécifiquement dans notre cas l'évolution spontanée de la maladie, ou les effets des traitements adjuvants, chez des patients ayant subi une exérèse carcinologique au niveau lingual.

De façon générale, cette recherche tâche, à partir d'analyses acousticoarticulatoires et perceptives, d'affiner nos connaissances du degré de flexibilité du système de production et de perception de la parole normale, mais surtout d'évaluer la perception et l'intelligibilité de l'articulation des 
consonnes et leur évolution, chez des patients ayant subi une exérèse carcinologique au niveau de la langue, pour mieux appréhender les possibles stratégies de compensation qu'un patient peut mettre en place après cette lourde opération.

Ces stratégies ont toutes comme but ultime chez le patient : atteindre une « cible » linguistique et maintenir une communication relativement normale et cohérente.

\section{Procédure expérimentale}

\subsection{Participants}

Nous avons passé le questionnaire d'auto-évaluation de la qualité de parole et du ressenti SHI (Speech Handicap Index) auprès de dix patients dans trois établissements hospitaliers alsaciens. Il s'agit plus précisément, de trois femmes (ZIM, PETR et HACH), et de sept hommes (SIB, SOM, GLAD, JCT, BIRL, ANT et ROJ). Ces patients ont subi différentes ablations linguales, partielles ou subtotales ( $c f$. Tableau1).

Tableau 1 : Répartition des exérèses et informations complémentaires concernant les cas cliniques étudiés (TNM : classification de la taille de la tumeur $(\mathrm{T})$, de la présence d'adénopathies $(\mathrm{N})$ et de la présence de métastases $(\mathrm{M}), \mathrm{M}$ : homme, $\mathrm{F}$ : femme, (hémiglossect : (hémi-) glossectomie, mandibulect : mandibulectomie, $\mathrm{G}$ : gauche, $\mathrm{D}:$ droite).

\begin{tabular}{|c|c|c|c|c|c|c|c|c|}
\hline ID Patients & Age & sexe & Profession & TNM & $T / d$ 'Exérèse & Reconstruction & $\mathrm{T} / \mathrm{comp}$ & Réeducation ortho \\
\hline SIB & 42 & M & Manager & T2NOMO & Glossect-Partielle G & Suture & Radiotherapie & Oui en libéral \\
\hline SOM & 30 & M & Ingénieur & TINOMO & Glossect-Partielle D & suture & Curiethérapie & Non \\
\hline ZIM & 69 & $\mathrm{~F}$ & Infirmière & PT 4 aN2bMO & Pelvi-Glossect D & suture & Radiotherapie & Non \\
\hline GLAD & 53 & M & Conducteur & T2NOMO & Glossect-Partielle D & Suture & Radiotherapie & Oui en libéral \\
\hline PETR & 65 & $\mathrm{~F}$ & Retraité & TANOMO & Pelvi-Glossect D & suture & Radiotherapie & oui au service ORL \\
\hline JCT & 53 & M & Manager & TANOMO & $\begin{array}{c}\text { Pelvi-Glossect - } \\
\text { Totale }\end{array}$ & $\begin{array}{c}\text { Lambeau } \\
\text { Antérolatéral }\end{array}$ & Radiotherapie & Oui en libéral \\
\hline $\mathrm{HACH}$ & 24 & $\mathrm{~F}$ & Etudiante & $\mathrm{T} 4 \mathrm{~N} 2 \mathrm{bMO}$ & $\begin{array}{c}\text { Hémi-Glossectomie } \\
\text { D }\end{array}$ & $\begin{array}{l}\text { Lambeau } \\
\text { Antébrachíal }\end{array}$ & Radiotherapie & Oui en libéral \\
\hline BIRL & 47 & M & Fonctionnaire & T3NOMO & $\begin{array}{l}\text { Pelvi-Glosso- } \\
\text { mandib G }\end{array}$ & Lambeau péroné & Radiotherapie & Oui en libéral \\
\hline ANT & 68 & M & Sans & T4N2bM0 & $\begin{array}{c}\text { Oro- } \\
\text { pharyngectomie } D\end{array}$ & Lambau peaucien & Radiotherapie & Non \\
\hline ROJ & 60 & M & Fonctionnaire & TINOMO & Glossect-Partielle D & Suture & Radiotherapie & Non \\
\hline
\end{tabular}

\subsection{Outil d'auto-évaluation de la qualité de parole et du ressenti (SHI)}

Le questionnaire utilisé pour auto-évaluer la qualité de la parole et le ressenti est le SHI (Speech Handicap Index de Rinkel et al. 2008), traduit en français par Degroote et al. (2012) et adapté du VHI (Voice Handicap Index de Jacobson et al., 1997). Le VHI a été créé dans le but d'évaluer l'aspect « invalidant» qu'un trouble vocal peut entrainer sur la qualité de vie, et de mesurer les conséquences psychosociales des troubles vocaux. 
La version du SHI utilisée comporte 30 items. Ces items se regroupent en deux catégories liées aux aspects psychosociaux (Ps) et à la parole (S). L'échelle de cotation subjective est établie sur 5 points (0-4), 0 correspondant à " jamais de gêne », 1 à "presque jamais gêné », 2 à " parfois gêné », 3 à " presque toujours gêné » et 4 à "toujours gêné 》). La passation du questionnaire d'auto-évaluation du ressenti (SHI) auprès des patients glossectomisés a eu lieu à différentes phases : Préop, soit avant l'opération, entre 1 mois et 1.5 mois après l'intervention chirurgicale (Post-Op1), à 3 mois et après tout traitements complémentaires (Post-Op2), à 6 mois (Post-Op3), etc.

Les sous-totaux obtenus à chaque phase ont été additionnés afin d'obtenir un score global sur 120. Plus le score est proche de 120, plus le handicap est élevé.

\section{Mesures}

En ce qui concerne les mesures, nous avons dans un premier temps calculé les scores totaux de la parole (S) et de l'aspect psychosocial (PS) de chaque patient. Par la suite, nous avons additionné les scores totaux Parole (S) et l'aspect Psychosocial (PS).

\subsection{Analyse des données}

Nous avons réalisé une analyse subjective des variations perceptives des réponses recueillies auprès des sujets pathologiques, lors de la passation du questionnaire d'autoévaluation de la qualité de parole et du ressenti (SHI), en comparaison aux réponses relevées auprès de ces mêmes sujets pathologiques, sur plusieurs phases pré et postopératoires.

Pour l'analyse statistique, nous avons utilisé le Test de Friedman qui consiste en une alternative non-paramétrique à l'analyse de variance, afin de voir l'effet du facteur phase (sessions de passation du SHI) sur les variables Score total, parole (S), l'aspect psychosocial (Ps).

\section{Hypothèses}

(1). Pour certains de nos patients, il est attendu une augmentation du score du handicap, qui serait due aux effets liés à la chirurgie et aux traitements complémentaires subis, à savoir la radiothérapie et la curiethérapie, lors des premières phases d'enregistrements postopératoires 1 et 2 .

(2). Étant donné que dans l'étude de Hamlet et al. (1990), la parole de patients souffrant d'un carcinome épidermoïde au niveau de la langue, classée T2 ou T3, a été jugée dans sa globalité normale, et que l'articulation serait préservée en phase Préopératoire, nous faisons l'hypothèse que le score du handicap et le score total au niveau de la perception de sa parole se situeraient entre 0 et 20, en phase Préopératoire.

(3). Nous pensons constater une récupération au niveau de la production de la parole et du ressenti des patients, lors des dernières phases qui se manifesteraient par une diminution du score, en rapport avec le délai opératoire et la rééducation orthophonique.

(4). Nous pensons que le facteur "sessions » de passation du (SHI) aurait un effet significatif sur les scores totaux, les scores totaux parole (S) et l'aspect psychosocial (PS) des patients.

En effet, ces deux dernières hypothèses sont motivées par la capacité des patients, de façon générale, à réajuster leurs stratégies articulatori-acoustiques, suite à la perturbation provoquée par la chirurgie reconstructrice. 


\section{Résultats}

Nous rappelons que pour l'étude de l'auto-évaluation de la qualité de parole et du ressenti (SHI), les résultats présentés infra sont constitués à partir de :

- $\quad$ scores obtenus lors de la passation du questionnaire (SHI) ;

- 7 patients ayant pu réaliser la tâche des 4 phases d'enregistrement ;

- les phases d'enregistrements et de passation du questionnaire d'autoévaluation de la qualité de parole et du ressenti pour les 2 patients (HACH et ROJ) sont au nombre de 2 , et pour le patient (ANT) sont au nombre de 3 Phases ;

- un total de 35 questionnaires (SHI).

Le tableau ci-dessous récapitule les scores calculés à partir des réponses de chaque patient (cf. Tableau 2).

Tableau 2 : L'ensemble des résultats au questionnaire d'auto-évaluation de la qualité de la Parole et $\mathrm{du}$ ressenti $(\mathrm{SHI})$, de tous les patients. $(\mathrm{S})=$ score parole, $(\mathrm{Ps})=$ score aspect psychosocial, $(\mathrm{QI})=$ score question indépendante 


\begin{tabular}{|c|c|c|c|c|}
\hline \multicolumn{5}{|c|}{ Speech handicap Index (SHI) } \\
\hline $\begin{array}{c}\text { Identification } \\
\text { des patients }\end{array}$ & Sessions & $(S)=$ speech & (Ps) = Psychosocial & Total \\
\hline PETR & Préop & 0 & 0 & 0 \\
\hline PETR & Postop1 & 14 & 0 & 14 \\
\hline PETR & Postop2 & 10 & 2 & 16 \\
\hline PETR & Postop3 & 4 & 1 & 7 \\
\hline ZIM & Préop & 3 & 0 & 3 \\
\hline ZIM & Postop1 & 12 & 15 & 31 \\
\hline ZIM & Postop2 & 14 & 13 & 28 \\
\hline ZIM & Postop3 & 8 & 11 & 21 \\
\hline SIB & Préop & 2 & 0 & 2 \\
\hline SIB & Postop1 & 23 & 12 & 35 \\
\hline SIB & Postop2 & 16 & 5 & 25 \\
\hline SIB & Postop3 & 3 & 0 & 3 \\
\hline BIRL & Préop & 10 & 2 & 13 \\
\hline BIRL & Postop1 & 17 & 14 & 34 \\
\hline BIRL & Postop2 & 12 & 13 & 26 \\
\hline BIRL & Postop3 & 14 & 12 & 29 \\
\hline GLAD & Préop & 5 & 0 & 5 \\
\hline GLAD & Postop1 & 19 & 7 & 28 \\
\hline GLAD & Postop2 & 12 & 0 & 14 \\
\hline GLAD & Postop3 & 5 & 0 & 6 \\
\hline SOM & Préop & 26 & 22 & 48 \\
\hline SOM & Postop1 & 29 & 22 & 53 \\
\hline SOM & Postop2 & 27 & 20 & 49 \\
\hline SOM & Postop3 & 20 & 19 & 31 \\
\hline JCT & Préop & 21 & 18 & 43 \\
\hline $\mathrm{JCT}$ & Postop1 & 52 & 48 & 100 \\
\hline JCT & Postop2 & 43 & 40 & 83 \\
\hline JCT & Postop3 & 30 & 34 & 72 \\
\hline ROJ & Préop & 2 & 0 & 2 \\
\hline ROJ & Postop1 & 25 & 25 & 50 \\
\hline $\mathrm{HACH}$ & Préop & 2 & 0 & 2 \\
\hline $\mathrm{HACH}$ & Postop1 & 31 & 16 & 51 \\
\hline ANT & Préop & 20 & 17 & 37 \\
\hline ANT & Postop1 & 34 & 25 & 59 \\
\hline ANT & Postop2 & 34 & 28 & 62 \\
\hline
\end{tabular}

Nous exposons ici les résultats du questionnaire d'auto-évaluation de la qualité de parole, pour le score Total, pour l'ensemble des patients. Notons que les scores des questions indépendantes (QI) ont été directement comptabilisés dans le score total.

En effet, en observant de près la Figure 1, nous avons constaté que les scores totaux sont plus élevés en Post-Op1 par rapport au Préop, ce constat étant valable pour l'ensemble des patients. En Post-Op1, le score total maximum atteint est de (100) pour le patient (JCT). Nous pourrions penser qu'il s'agit là d'un score excessif et que le patient surestimerait son état. Cependant, étant donné que le patient (JCT) a subi une pelvi-glossectomie totale suivie d'une reconstruction, nous pouvons imaginer qu'une telle exérèse ne pourrait que rendre compte des lourdes répercussions de ces interventions sur la qualité de vie du patient. Il est vrai que les trois fonctions vitales du patient ont été affectées, à savoir : la mastication, la 
déglutition, la qualité de parole et le ressenti, en plus des dégradations de l'image de soi et de l'aspect psychosocial.

Toujours en Post-op1, nous avons relevé le score total le plus bas qui est de (3) pour la patiente (PETR), une patiente ayant subi une pelvi-glossectomie partielle classée T4. Les scores totaux des autres patients, se situent entre les scores des deux patients cités supra (JCT et PETR). Nous avons constaté que pour les patientes (ZIM et PETR), les scores totaux en Post-Op1 sont de (31 et 14), respectivement. Rappelons que les patientes (ZIM et PETR) ont subi la même la prise en charge chirurgicale et thérapeutique (pelviglossectomie plus radiothérapie). Ceci dit, la qualité de parole et du ressenti pour ces deux mêmes patientes est plus altérée chez la patiente ZIM que PETR et cela malgré la similitude $\mathrm{du}$ traitement subi. Pour les quatre patients ayant subi une glossectomie partielle (SOM, GLAD, SIB, et ROJ), nous avons observé une différence au niveau des scores totaux, déjà en Préop, les scores étant de (48) pour le patient SOM et de (2) pour les trois autres patients (GLAD, SIB, et ROJ). En effet, nous pouvons voir que les scores totaux des patients (GLAD, SIB et ROJ) sont similaires. Cependant, celui de SOM reste assez élevé pour une auto-évaluation de la qualité de parole et du ressenti avant l'acte chirurgical. Cela semble montrer une sur-appréciation Préopératoire de l'état du patient. En Post-Op1, une légère augmentation des scores totaux a été observée pour les patients SIB et SOM (35 et 53 respectivement), contrairement à ce qui a été relevé chez les patients GLAD et ROJ (28 et 50 respectivement) (cf. Tableau 2).

Pour les autres patients ayant subi différentes ablations accompagnées de reconstructions, nous avons observé qu'en Préop la patiente $\mathrm{HACH}$ (2) représente le score le plus bas par rapport aux trois autres patients (JCT, BIRL et ANT) qui ont comme score (43, 13 et 37 respectivement). En Post-Op1, les scores totaux augmentent brusquement (51) pour $\mathrm{HACH}$, puis $(100,34$ et 59) pour (JCT, BIRL et ANT) respectivement. Ces résultats peuvent s'expliquer par la sévérité du handicap engendrée par les conséquences propres à chaque traitement chirurgical affectant globalement la qualité de la parole, la qualité de vie et du ressenti.

En Post-Op2 nous avons remarqué que les scores totaux ont tendance à baisser dans le cas des patients (SIB, JCT, SOM, GLAD et BIRL), ou à se stabiliser et à se régulariser ; c'est le cas de la patiente (ZIM). Les deux seuls patients pour qui le score total de la qualité de parole et du ressenti a légèrement grimpé sont les patients (PETR et ANT).

En Post-Op3, quasiment tous les scores totaux ont baissé chez tous nos patients en se rapprochant graduellement des scores relevés en Préop, mais sans pour autant les atteindre. Seul le score total du patient (GLAD) a pu rejoindre le score initialement perçu en Préop.

Le score total (SHI) le plus élevé en Post-Op3 reste celui du patient JCT. Notons que pour ce patient (JCT), en plus des répercussions sur la parole et l'aspect psychosocial, il s'alimente par le biais d'une sonde gastrique et souffre de xérostomie (sècheresse de la cavité buccale), raison pour laquelle le score total de ce patient resterait assez remarquable, même en Post-Op3 ( $c f$. Figure 1). 


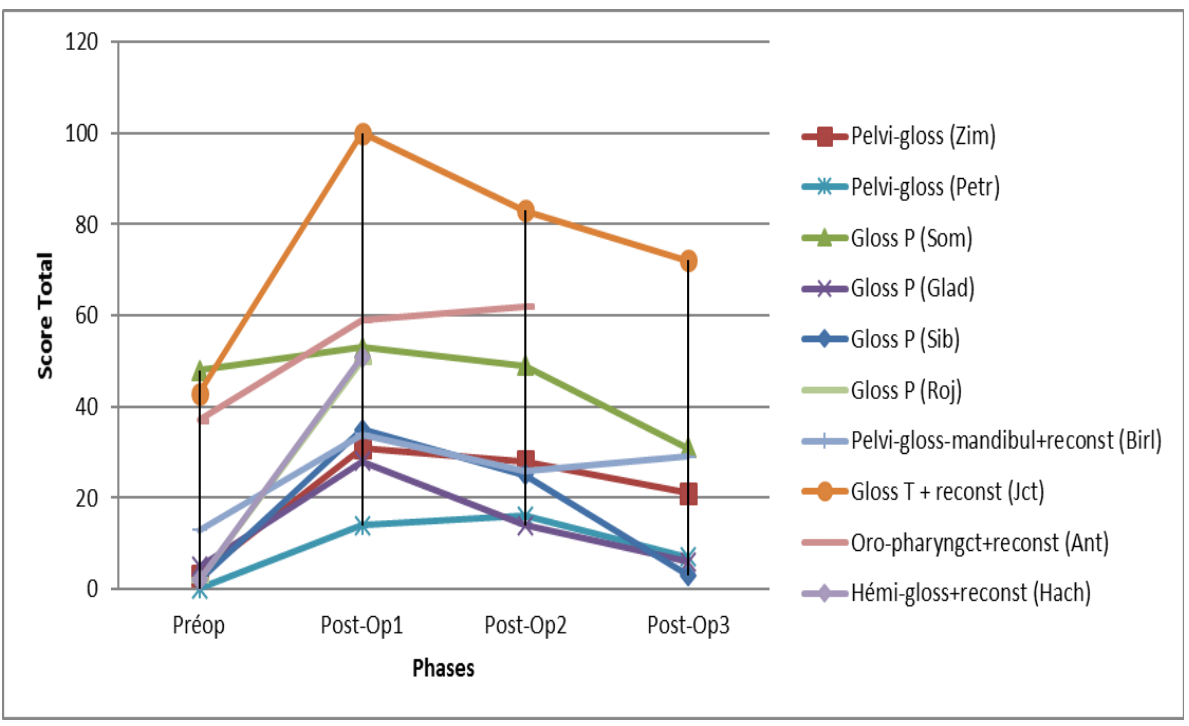

Figure 1 : Résultats des auto-évaluations de la qualité de parole, pour le score Total, pour l'ensemble des patients, à partir du questionnaire SHI (Speech Handicap Index)

En ce qui concerne les résultats du questionnaire d'auto-évaluation de la qualité de parole, pour les aspects liés à la parole et pour l'ensemble des patients, nous avons remarqué qu'en Préop, les scores de parole (S) se situent entre (0 et 10) sur un score total de 56, pour la plupart des patients, sauf pour (SOM, JCT et ANT), pour qui les scores démarrent à $(26,52$ et 34) respectivement, sur un score total de 56.

Ces autoévaluations sont probablement liées au volume de la tumeur qui était important. Nous avons observé que par rapport au Préop, les scores parole(S) augmentent en PostOp1. Les tendances des répartitions des scores parole (S) se comportent de façon quasi similaire à ce qui a été constaté pour les scores totaux. Comme nous pouvons le voir sur la ( $c f$. Figure 2), en Post-Op1 les scores parole (S) sont très élevés pour le patient (JCT) et très bas pour la patiente (ZIM).

Les scores parole (S) des autres patients se situent dans l'intervalle des scores de ces deux patients. Une augmentation est observée chez tous nos patients en Post-Op1. En Post$\mathrm{Op} 2$, après les traitements adjuvants, nous avons constaté une diminution des scores parole (S) pour tous les patients ; les scores parole $(\mathrm{S})$ ont tendance à se régulariser pour certains de nos patients et baisser jusqu'à rejoindre les scores relevés initialement en Préop pour d'autres. Hormis la patiente ZIM, pour qui le score parole a légèrement augmenté, nous pensons que cette augmentation des scores parole (S) serait liée aux effets secondaires de la radiothérapie, puisque cette patiente souffrait de brulures post-radiques qui lui provoquaient des aphtes et des cloques. Cela peut probablement engendrer une gêne lors de la production de la parole.

En Post-Op3, nous pouvons voir que les scores parole (S) reviennent à la normale pratiquement pour tous les patients, excepté le patient BIRL, patient ayant subi une pelviglosso-mandibulectomie pour qui le score parole (S) augmente légèrement. 


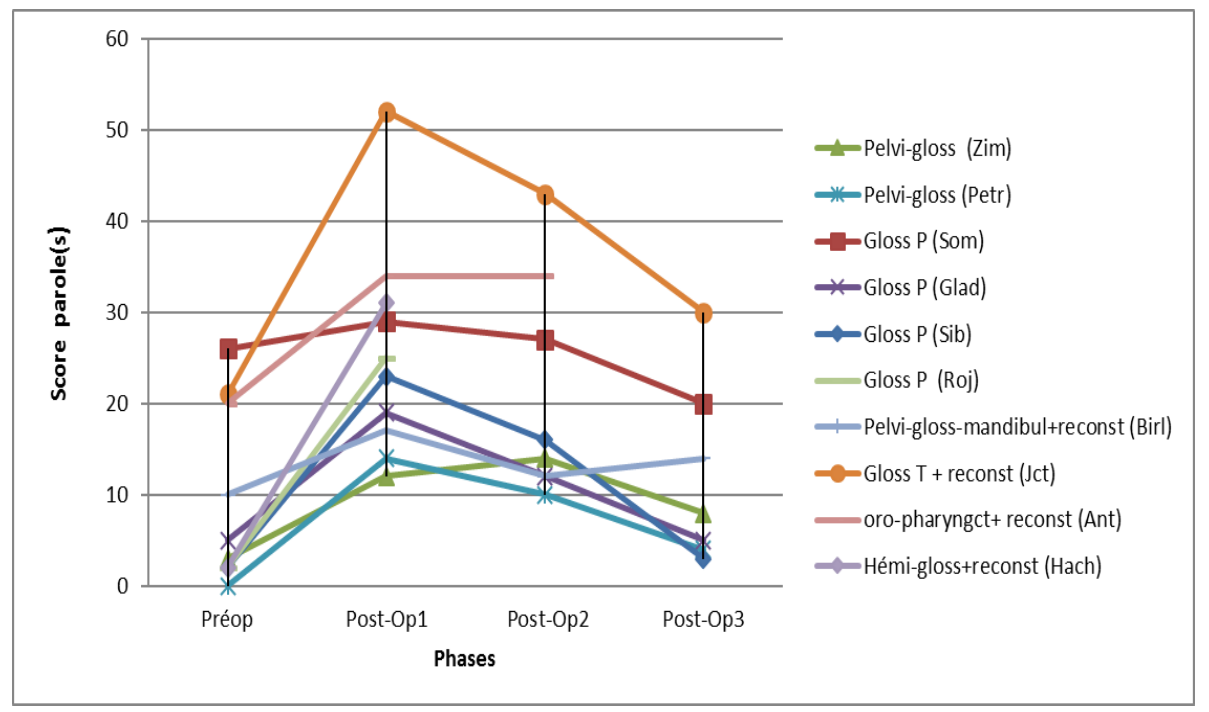

Figure 2 : Résultats des auto-évaluations de la qualité de parole, pour le score de parole, pour l'ensemble des patients, à partir du questionnaire SHI (Speech Handicap Index)

En ce qui concerne les résultats du questionnaire d'auto-évaluation de la qualité de parole et $\mathrm{du}$ ressenti pour les aspects psychosociaux, nous pouvons voir que pour les scores de l'ensemble des patients, les mêmes tendances que les résultats relevés sur les scores totaux et les scores parole (S) se retrouvent.

Le même scénario semble se reproduire ici. Nous pouvons voir que les scores de l'aspect psychosocial commencent à 0 chez plusieurs patients, pour augmenter en Post-Op1 chez l'ensemble de nos patients, hormis la patiente (PETR) pour laquelle le score (Ps) est resté à 0 . À peu de choses près, ce sont les mêmes patients qui ont atteint les plus haut scores (totaux et parole) également pour les scores (Ps), c'est-à-dire (SOM, ANT, JCT et ROJ), respectivement. En Post-Op2, nous avons constaté une baisse importante des scores chez quasiment tous nos patients, exceptés chez les mêmes patients les plus affectés à savoir : (JCT et ANT).

Enfin, en Post-Op3, les scores Ps rejoignent les valeurs initialement relevées en Préop pour les patients (GLAD, SIB et PETR), mais ce n'est pas le cas pour les autres patients ( $c f$. Figure 3). Globalement, le score de l'aspect psychosocial est plus élevé en Post-Op1 et a tendance à diminuer graduellement en Post-Op2 et 3. 


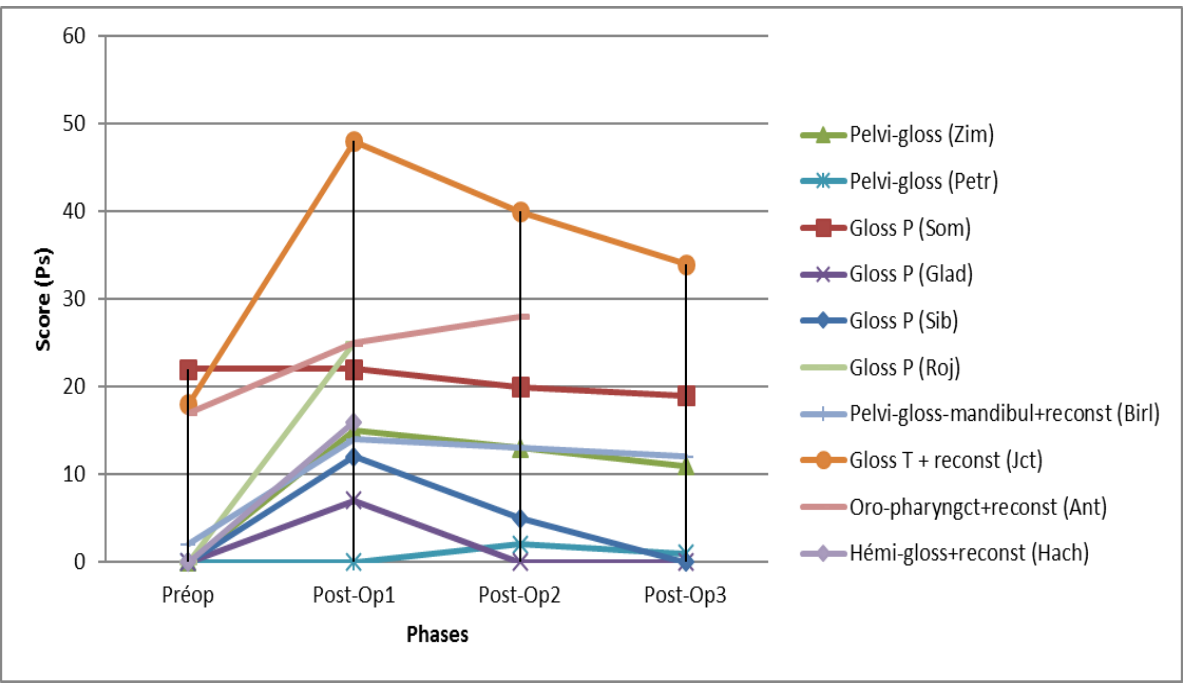

Figure 3 : Résultats des auto-évaluations de la qualité de parole, pour les aspects psychosociaux, pour l'ensemble des patients, à partir du questionnaire SHI (Speech Handicap Index)

\section{Analyse statistique de l'auto-évaluation de la qualité de parole et du ressenti (SHI)}

Nous avons réalisé, à partir de ces données, une analyse statistique des scores bruts obtenus à l'auto-évaluation de la qualité de parole et du ressenti pour les 7 patients ayant accompli la tâche des 4 sessions (phases) de passation et d'enregistrements (en utilisant le test de Friedman, à mesures répétées par rangs pour des échantillons appariés avec corrections Tukey). Nous avons appliqué l'ANOVA de transformation alignée (ART ANOVA) qui consiste en une approche non-paramétrique permettant l'analyse de multiples variables indépendantes, des interactions pour des mesures répétées (Mangiafico, 2016).

L'analyse statistique a été réalisée à l'aide du logiciel R. Dans le cadre de cette analyse, nous avons étudié l'effet du facteur "session » ou phase de passation du questionnaire d'auto-évaluation de la qualité de parole et du ressenti (SHI) sur les covariances indépendantes, à savoir : «Score total, score parole, score psychosocial» (cf. Tableau 3). Les résultats montrent qu'il existe un effet significatif du facteur session (phase de passation), pour le score Total entre toutes les phases pré et post-opératoires $(p<0,05)$. En effet, nous avons observé que l'analyse qualitative du score total indique qu'il subsiste un accroissement du handicap en post-opératoire 1 et 2 , par rapport à la phase Préopératoire. Avec le temps, une amélioration est constatée entre les phases Post-Op 1 et 3. Pour le score total, la différence entre les scores est significative entre les sessions Préop et Post-Op1, Préop et Post-Op2, Post-Op1 et Post-Op3, Post-Op2 et Post-Op3 (à p<0.05) (cf. Tableau 3)

Les résultats de l'analyse statistique, illustrés par le Box Plot ci-dessous ( $c f$. Figure 4), montrent des différences au niveau des scores totaux à travers les phases pré et post chirurgicales; ces différences sont significatives. Nous avons également constaté deux valeurs anormales qui correspondent aux scores totaux du patient JCT, en Post-Op1 et en Post-Op3. 


\section{Score total}

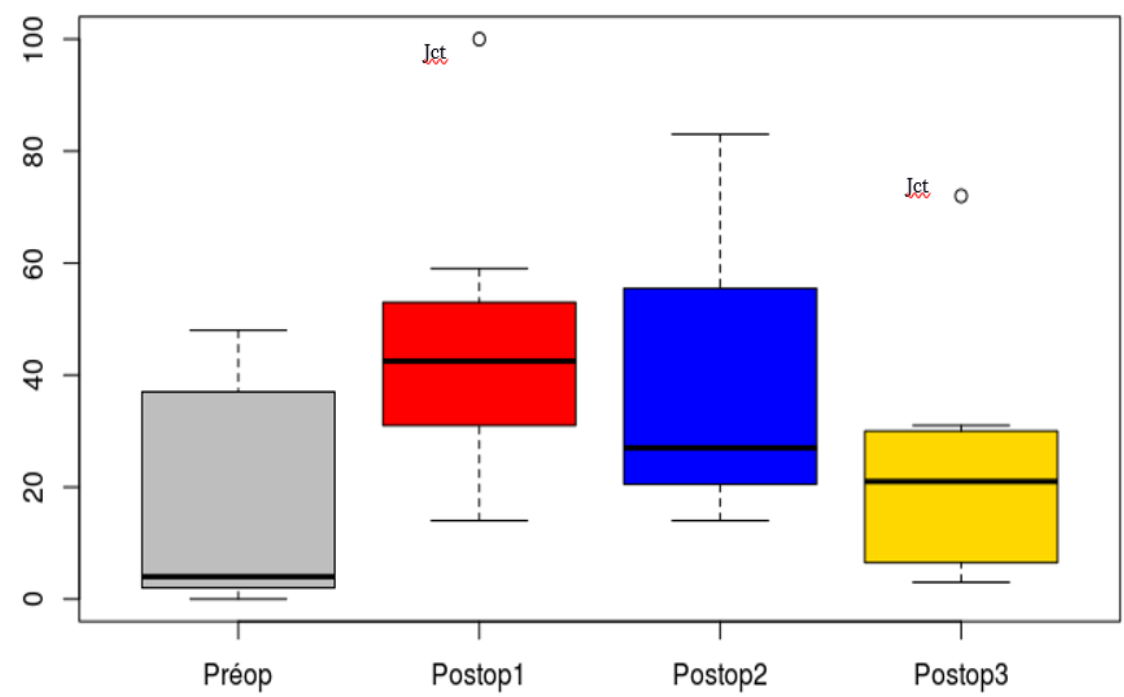

Figure 4 : Représentation par Box plot des scores totaux à travers les sessions de passation du SHI (Speech Handicap Index)

Pour le score parole (S), nous avons relevé des différences significatives entre toutes les phases pré et post-chirurgicales $(\mathrm{p}<0,05)$, excepté entre la phase Préop et Post-Op3, PostOp1 et Post-Op2, où la différence des scores parole n'étaient pas significative $(\mathrm{p}>0,05)$.

Les résultats de l'analyse statistique, représentés dans le Box Plot ci-dessous ( $c f$. Figure 5), montrent des différences au niveau des scores parole, à travers les phases pré et postchirurgicales, ces différences étant significatives.

\section{Score parole}

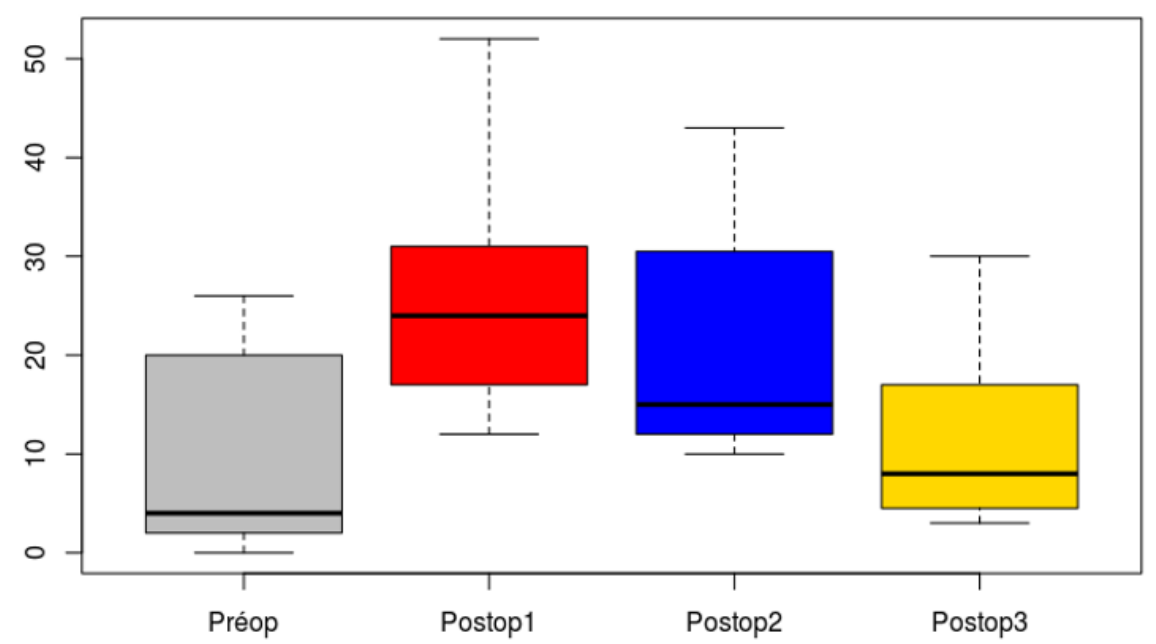


Figure 5 : Représentation par Box plot du score parole à travers les sessions de passation du SHI (Speech Handicap Index)

En ce qui concerne le score psychosocial, nous avons identifié des différences significatives entre les phases Préop et Post-Op1, Préop et Post-Op2, Post-Op1 et Post-Op3 $(\mathrm{p}<0,05)(c f$. Tableau 3).

Les scores psychosociaux n'étaient pas significatifs entre le Préop et Post-Op3 $(\mathrm{p}>0,05)$, signe d'une récupération et d'une diminution de la sévérité du handicap, puisque les scores se rapprochent ou atteignent les scores initialement relevés en Préop ( $c f$. Tableau 2).

Nous avons également observé une valeur anormale qui correspond aux scores de l'aspect psychosocial du patient JCT, en Post-Op1 ( $c f$. Figure 6). Nous avons identifié des différences entre les phases Post-Op1 et Post-Op2, Post-Op2 et Post-Op3 pour le score psychosocial, mais ces différences ne sont pas significatives sur le plan statistique.

\section{Psychosocial}

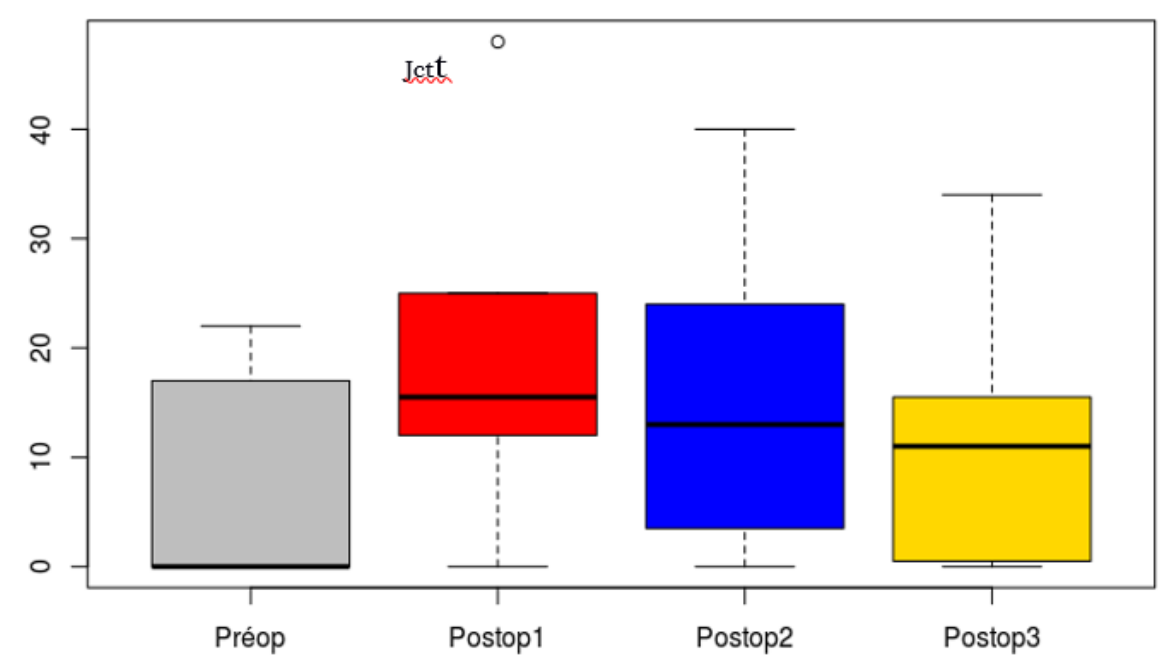

Figure 6 : Représentation par Box plot du score de l'aspect psychosocial à travers les sessions de passation du SHI (Speech Handicap Index)

L'augmentation des scores du handicap est largement liée à la chirurgie et aux traitements adjuvants. En effet, à travers les différents cas des patients sujets de notre étude, nous pouvons voir que plus le volume de la tumeur est important, plus la taille de la résection est large. Cela a une incidence directe sur le degré et la sévérité du handicap. Globalement, lors de la phase Préopératoire, les scores du handicap se situent entre 0 et 48 . Avec le délai opératoire et la rééducation orthophonique pour certains de nos patients, les scores ont tendance à baisser. Nous considérons cela comme le signe d'une récupération et d'une amélioration de la qualité de parole et du ressenti, en Post-Op3. La rééducation et le facteur temps ont un effet important sur la récupération et la régularisation des scores psychosociaux (Ps), parole (S) et les scores totaux des patients. 
Tableau 3 : Résultats statistiques à partir du questionnaire d'auto-évaluation de la qualité de parole et du ressenti (Test de Friedmann)

\begin{tabular}{|l|l|l|l|l|l|}
\hline Covariances/Phases & Préop-Post-0p1 & Préop-Post-0p2 & Préop-Post-0p3 & Post-0p1- Post-0p2 & Post-0p2- Post-0p3 \\
\hline Score total & $\mathrm{P}=1,6^{\mathrm{e}-07}$ & $\mathrm{P}=3,2^{\mathrm{e}-05}$ & $\mathrm{P}=0,053$ & $\mathrm{P}=3,2^{\mathrm{e}-05}$ & $\mathrm{P}=0,018$ \\
\hline Score parole (S) & $\mathrm{P}=2,8^{\mathrm{e}-07}$ & $\mathrm{P}=5,9^{\mathrm{e}-05}$ & $\mathrm{P}=0,056$ & $\mathrm{P}=2,2^{\mathrm{e}-05}$ & $\mathrm{P}=0,011$ \\
\hline Score psychosocial (PS) & $\mathrm{P}=0,00072$ & $\mathrm{P}=0,03912$ & $\mathrm{P}=1,00000$ & $\mathrm{P}=0,00352$ & $\mathrm{P}=0,1816$ \\
\hline
\end{tabular}

En effet, plus la taille de la résection est importante, plus le score parole est élevé, plus le handicap est sévère. À titre d'exemple, nous pouvons voir sur le graphique supra (cf. Figure 5) que le patient ayant subi une pelvi-glossectomie totale (JCT) a atteint le score maximum. Une diminution du score parole est observable en Post-Op2, signe d'une amélioration de la qualité de parole et du ressenti, pour atteindre en Post-Op3, les scores de départ relevés en Préop.

\section{Conclusion}

Dans cette étude nous avons traité les réponses aux questionnaires d'auto évaluation de la qualité de parole et du ressenti (SHI). Les résultats du questionnaire d'auto évaluation de la qualité de la parole et du ressenti nous ont permis de caractériser le degré de sévérité du handicap pour chaque patient. Ces résultats montrent que, globalement, l'évaluation de la qualité de la parole et du ressenti corroborent le diagnostic de l'examen ORL postopératoire. L'altération et la dégradation sont omniprésentes à travers l'évaluation de la qualité de parole et du ressenti, en phase post-op1. En effet, chaque patient apprécie bien son état selon le type de prise en charge chirurgicale et thérapeutique qu'il a subi. Nous avons pu voir que l'estimation des séquelles, après une exérèse carcinologique localisée dans le système de production de la parole, est en adéquation avec les scores totaux en postop1.

En Post-Op2, les réponses des patients témoignent d'une amélioration de la qualité de parole et du ressenti, à l'instar d'une diminution des scores totaux, parole (S) et psychosocial (PS). Nous pouvons imaginer que la qualité de parole et du ressenti relevés auprès d'un patient ayant subi une glossectomie partielle sont moins importants que chez un patient qui a subi une pelvi-glossectomie partielle ou totale suivie d'une reconstruction.

Une progression dans l'amélioration de la qualité de vie et du ressenti a été remarquée en Post-Op3. Cette amélioration peut être liée au temps pour certains patients et à la rééducation orthophonique pour d'autres. Les résultats des analyses statistiques effectuées pour chaque score confirment ces conclusions.

De manière générale, nos résultats peuvent être rationalisés dans le cadre de la Théorie de la Viabilité (Aubin \& Cellina, 1984), car les réajustements effectués par les patients demeurent un ensemble de trajectoires viables qui permettent une émergence cohérente d'un système linguistique, pouvant atteindre, en l'occurrence ici, les « cibles » aérodynamiques, articulatoires et acoustiques recherchées (Sock \& Vaxelaire, 2001), qui seront alors perçues correctement en auto-évaluation. 
Nous avons vu que les modifications anatomiques de la cavité buccale entraînent des perturbations plus ou moins importantes qui affectent l'intelligibilité de la parole. Les nouvelles productions, qui sont parfois déviantes par rapport à des productions typiques, peuvent contribuer à révéler les limites du domaine de la viabilité des entités linguistiques (sons, syllabes, mots, etc.) en production-perception de la parole. Chaque locuteur, à partir de son système de production de la parole, est capable de mettre en place des ajustements qui lui sont propres, que ce soit un locuteur sain ou pathologique. Cependant, ce sont les locuteurs pathologiques qui ont plus tendance à recourir à des manœuvres compensatoires pour produire une parole acceptable. Ainsi, leurs données témoignent de l'existence de zones distinctes et propres à chacun de ces locuteurs. La réalisation des gestes articulatoires est, en revanche, bornée dans ces zones par certaines contraintes (dans la mesure où l'intégrité du message doit être conservée) afin que l'émergence du son soit viable.

Il nous semble primordial de signaler ici que le concept de la viabilité en production de la parole est en relation directe non seulement avec la notion du maintien des oppositions phonologiques, mais aussi avec les trajectoires et les configurations adoptées par des catégories articulatori-acoustiques. En effet, ces relations interviennent sur le plan temporel et fréquentiel, en contexte de perturbations dites internes ou externes des systèmes phonologiques. Aussi, les catégories articulatori-acoustiques produites par un locuteur pathologique peuvent varier entre entités significativement modifiées et entités relativement résistantes, évoluant à l'intérieur de zones dites viables pour assurer l'émergence d'un message linguistique compréhensible.

Au total, comme l'indique Bechet (2011), la Théorie de la Viabilité en productionperception de la parole révèle que plusieurs solutions articulatoires peuvent mener à une même "cible » acoustique à condition que ces solutions soient situées à l'intérieur de la zone de viabilité qui sert de " garde-fou » à l'émergence correcte d'un son. Pareillement, de nombreuses solutions acoustiques peuvent permettre d'atteindre une «cible » perceptive équivalente, mise au jour dans cette étude par le SHI.

De ce fait, la variabilité est observable dans le système de production-perception de la parole, aussi bien au niveau articulatoire qu'acoustique, aboutissant au déploiement de multiples stratégies éventuelles réalisables par les locuteurs. Ce constat est particulièrement important pour comprendre certaines données issues de productions pathologiques en parole. On sait aussi que des réajustements sont possibles à différentes phases chez les locuteurs pathologiques, afin d'atteindre des cibles acoustiques et perceptives comparables à celles relevées initialement auprès d'un même locuteur pathologique (avant une perturbation quelconque), ou bien auprès des locuteurs sains ne montrant aucun dysfonctionnement au niveau de leur système de production-perception de la parole.

Rappelons qu'en nous s'intéressant à l'étude de la parole pathologique, nous nous plaçons dans le paradigme qui pose qu'il est possible de tirer des informations sur la production de la parole normale à partir de données pathologiques atypiques. Potentiellement, la production de la parole après glossectomie donnerait la possibilité d'observer, de manière générale, la variabilité en production de la parole. Il a donc été possible, dans cette étude, de discerner la variabilité et son étendue, mais aussi d'en déterminer ses limites (voir les données du patient JCT, par ex.). 


\section{Références bibliographiques}

Acher, A., Sato, M., Lamalle, L., Vilain, C., Savariaux, C., Gerber, S., Attye, A., Krainik, A., Bettega, G., Righini, C.A., others. (2014). Evolution des activations cérébrales lors de la production de parole après exérèse au niveau de la cavité orale. In XXX ${ }^{\text {ièmes }}$ Journées d'Etudes Sur La Parole (JEP2014).

Aubin, J.-P. Cellina A., (1984). Differential inclusions. New York : Springer Verlag.

Bechet (2011). Perturbation de la production des occlusives chez des locuteurs présentant une division palatine ou labio-palatine (Thèse de doctorat). Université de Strasbourg.

Bjordal K., Hammerlid E., Ahlner-Elmqvist M. (1999). Quality of life in head and neack cancers patients: validation of the European Organization for Research and Treatment of Cancer quality of life questionnaire-H\&N35. J Clin Oncol 17,1008-1019

Calmet-Smadja, M. Crevier-Buchman, L. Tessier, C. (2003). Évaluation de la qualité de vie après glossectomie partielle : étude des corrélations entre la qualité de vie, l'évaluation fonctionnelle et l'intelligibilité de la parole. Paris, France.

Degroote, G.,Simon ,J.,Borel,S.,\& Crevier-Buchman L. (2012). The French version of the Speech Handicap Index: validation \& comparison with the Voice Handicap Index. Folia Phoniatrica et Logopaedica 64, (1), 20-25.

Gritz, ER, Vernon, SW, Peterson, SK, Baile, WF, Marani, SK, Amos, CI, Frazier, ML, Lynch, PM., (1999). Distress in the cancer patient and its association with genetic testing and counseling for hereditary non-polyposis colon cancer. Cancer Res Ther Control 8, 35-49

Jacobson,B. $\quad$ H.,Johnson,A.,Grywalski ，C.,Silbergleit ，A.,Jacobson,G.,Benninger,M. S., ～\& Newman ,C. W (1997). The Voice Handicap Index (VHI): Development and validation. American Journal of Speech Language Pathology 6, (3), 66-70.

Hammerlid, E., Ahlner-Elmqvist, M., Bjordal, K., Biörklund, A., Evensen, J., Boysen, M., Jannert, M., Kaasa, S., Sullivan, M., Westin, T. (1999). A prospective multicentre study in Sweden and Norway of mental distress and psychiatric morbidity in head and neck cancer patients. British Journal of Cancer, 80, 766-774

Hamlet S.L., Mathog R.H., Patterson R.L. (1990). Tongue mobility in speech after partial glossectomy. Head and Neck, 12, (3), 210-217.

Korpijaakko-Huuhka A.-M., Söderholm A.-L. et al. (1998). Long-lasting speech and oral motor deficiencies following oral cancer surgery: a retrospective study. Logopedics Phoniatrics Vocology, 24 (3), 97-106.

Fauvet, F., Schultz P., Debry C., Hirsch F., Sock R. (2006) : Intelligibilité de la parole après glossotomie totale et réhabilitation orthophonique précoce, in Actes des XXVI èmes. Journées d'Étude sur la Parole, 451-454, Dinard.

Mangiafico, S.S. 2016. Summary and Analysis of Extension Program Evaluation in R, version 1.18.1

Rinkel, R. N.,Verdonck- De Leeuw,I. M.,Vanreij,E. J.,A Aronson ,N. K.,\& Leemans, C. R (2008). Speech Handicap Index in patients with oral and pharyngeal cancer: better understanding of 
patients' complaints. Head and Neck 30, (7), 868-874.

Savariaux, C., Perrier, P., Pape, D., Lebeau, J. (2001). Speech production after glossectomy and reconstructive lingual surgery: a longitudinal study. In Proceedings of the 2nd International Workshop on Models and Analysis of Vocal Emissions for Biomedical Applications (MAVEBA). Firenze, Italy.

Sock, R., \& Vaxelaire, B. (2001). Réflexions sur le timing de la quantité. Travaux de l'Institut de Phonétique de Strasbourg, TIPS, 31, 89-126.

Sock, R. (1998). Organisation temporelle en production de la parole. Émergence de catégories sensori-motrices phonétiques. (Doctorat d'état). Université de Grenoble.

Vaxelaire, B. (2006) La résistivité spatio-temporelle des gestes linguistiques. Ou perturber la linguistique en augmentant la vitesse d'élocution. In Perturbations et réajustements : langue et langage. Vaxelaire B. Sock R. Kleiber G. Marsac F. (eds.). Publications de l'Université Marc Bloch - Strasbourg. 